\title{
Um Modelo Espacial de Demanda Habitacional para a Cidade do Recife ${ }^{\star}$
}

\author{
- Rubens Alves Dantas* - André Matos Magalhães**

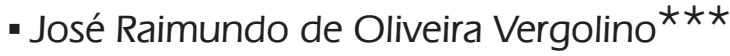

\begin{abstract}
Resumo
Este trabalho mostra a importância da utilização da econometria espacial nos estudos dos fenômenos relacionados à economia urbana, em particular, no comportamento do mercado habitacional. Nas análises realizadas, com o objetivo de estimar uma Função de Demanda por Habitação para a cidade do Recife, com base em informações do Censo Demográfico (2000) e dados de imóveis financiados pela Caixa Econômica Federal, verificaram-se fortes indícios de dependência espacial em todas as variáveis econômicas exploradas. Verifica-se que a maneira de considerar a questão espacial, em função de distâncias da habitação a polos de influência ou dividindo o espaço em regiões, como vem ocorrendo corriqueiramente na literatura, não é capaz de explicar completamente o comportamento da demanda por habitação. Os resultados indicam que as equações de demanda tradicionalmente estimadas, sem levar em conta os efeitos de dependência espacial, podem gerar resultados tendenciosos, com a elasticidade-preço representando menos de 50\% da estimativa do modelo espacial.
\end{abstract}

\section{Palavras-Chave}

demanda por habitação, econometria espacial

\begin{abstract}
This paper shows the importance of using spatial econometrics in the studies related to the analysis of housing market's behavior. In empirical analysis carried out on the object of estimating a Housing Demand Function for the city of Recife, Brazil, based in information from the Demographic Census (2000) and data from real state financed by Caixa Econômica Federal, strong indications of spatial dependency were verified in all explored economical variables. One verifies that the manner of considering the spatial issue as a function of distances of housing to influence poles or space division in regions, like currently occurring in literature, is not capable of explaining entirely the behavior of the housing demand. The results indicate that the demand equations traditionally estimated, without considering the effects of spatial dependency may generate biased results, where the price-elasticity by the traditional model represents less than $50 \%$ of the estimates of the spatial model.
\end{abstract}

\section{Keywords}

housing demand, spatial econometrics

\section{JEL Classification}

$\mathrm{R} 21, \mathrm{C} 21$

+ Os autores agradecem o suporte financeiro do CNPq. Os autores também são gratos aos participantes do encontros da RSAI 2008 e da ABER 2008 pelos comentários e críticas. Erros e omissões são de responsabilidade exclusiva dos autores.

* Departamento de Engenharia Civil, Universidade Federal de Pernambuco (UFPE) e Escola Politécnica de Pernambuco (POLI-UPE). E-mail: rubens@dantas.eng.br.

** Departamento de Economia, Universidade Federal de Pernambuco (UFPE) e Regional Economics Applications Laboratory (REAL) University of Illinois, Urbana, Illinois, USA. E-mail: magalhaes@upfe.br.

$\star \star \star$ Faculdade Boa Viagem (FBV). E-mail: jose.vergolino@globo.com.

Endereço para contato: Departamento de Economia - UFPE - Av. dos Economistas - Cid. Universitária - Recife - PE. CEP: CEP: 50740-590.

(Recebido em maio de 2009. Aceito para publicação em abril de 2010). 


\section{Introdução}

O mercado habitacional, tanto do ponto de vista de geração de empregos e renda para o sistema econômico, como também da importância social do produto habitação, constitui-se num setor-chave de qualquer economia. A compreensão do seu funcionamento é fundamental para elaboração e implementação de políticas habitacionais e urbanas. Para isto, torna-se necessária a estimação confiável da função de demanda por habitação. Os modelos teóricos voltados à compreensão do funcionamento do mercado habitacional podem ser divididos em dois grupos: macroeconômicos, que procuram verificar o comportamento da demanda de habitações de forma agregada, e microeconômicos, que estão direcionados para o estudo do comportamento individual do consumidor, isto é, modelos que explicam como o indivíduo toma sua decisão no processo de escolha da habitação. Embora ambos os grupos forneçam contribuições importantes para a compreensão dos diferentes aspectos relacionados ao mercado habitacional, a análise, neste trabalho, será de caráter microeconômico, posto que se pretende elaborar um modelo espacial de demanda por habitação. ${ }^{1}$

Em geral, a análise microeconômica do mercado habitacional é feita em dois estágios: no primeiro, estima-se a equação de preços hedônicos e, com base nestes resultados e mais as características sociodemográficas da família, estima-se a função de demanda por habitação. Pela equação de preços hedônicos, podem ser obtidos os preços implícitos ou hedônicos de cada característica individual da habitação, ${ }^{2}$ que refletem a utilidade que cada uma gera para o consumidor, como também o preço total que o mesmo está disposto a pagar pela cesta de serviços de habitação com que se defronta no mercado. A partir da função demanda por habitação, é possível estabelecer um melhor entendimento de como varia o consumo individual de habitação em relação a variações na renda familiar ou no preço da habitação, ou seja, o conhecimento das elasticidades-renda e preço da habitação.

As evidências disponíveis na literatura nacional indicam que muito pouco foi realizado, tanto a nível de modelos de preços hedônicos, como também em relação à estimação da função de demanda habitacional no Brasil. Em relação à estimação de modelos de preços hedônicos, em um dos primeiros trabalhos publicados, Dantas e Cordeiro (1988) analisam um segmento do mercado imobiliário de lotes urbanos, situados em três bairros do Recife; em seguida, Gonzalez e Formoso (1994) examinam uma base de dados em Porto Alegre; Aguirre e Faria (1996) fazem uma aplicação da metodologia a dados de São Paulo; Aguirre e Macedo (1996) estimam

1 Nos modelos macroeconômicos, é grande a perda de informações relevantes, principalmente em relação à localização da habitação, que é uma das variáveis mais importantes na formação dos preços de mercado.

2 Conforme metodologia definida por Rosen (1974). 
preços hedônicos para o Mercado Imobiliário de Belo Horizonte; Dantas e Cordeiro (2001) analisam o mercado de apartamentos na Região Metropolitana do Recife (RMR) e Hermann (2003) estima o preço implícito de amenidades urbanas no município de São Paulo. Em relação à estimação da função de demanda habitacional, a escassez é ainda maior. Na literatura pesquisada, apenas quatro trabalhos examinam o comportamento do mercado habitacional no Brasil: Lucena (1985) estima elasticidades-renda, usando curvas de Engel, e encontra resultados de 0,61 para São Paulo, 0,84 para Rio de Janeiro e Recife e 0,81 para o Brasil como um todo, mas reconhece que as medidas utilizadas para quantificar o consumo de habitação são muito incipientes; Balarine (1995) estuda o impacto de fatores socioeconômicos na formação do estoque habitacional em Porto Alegre, entre 1970 e 1991, numa visão macroeconômica, mas não chega a resultados explícitos sobre as elasticidades-renda e preço da habitação; Santos e Cruz (2000) usam dados agregados de série temporal anual no período 1977/1997 e fazem uma aplicação do modelo de cash-flow; ${ }^{3}$ e Biderman (2001) realiza uma análise da demanda e oferta no mercado de novos imóveis em São Paulo, mas também não chega a nenhum resultado explícito sobre as elasticidades-renda e preço da habitação. Estas deficiências podem ser atribuídas, em grande parte, às dificuldades na obtenção de dados. ${ }^{4}$

Observa-se que, em todos os trabalhos citados, a abordagem econométrica utilizada é a dos mínimos quadrados ordinários, ${ }^{5}$ utilizada para explicar o comportamento do mercado habitacional, onde a questão espacial é considerada tomando-se como referência distâncias a polos de influência ou setorização das cidades, admitindo-se a independência espacial da variável explicada e dos resíduos do modelo de regressão. Contudo, segundo Anselin (1988), dados associados à posição que ocupam no espaço (cidades, regiões, bairros ou a própria coordenada geográfica), como é o caso da habitação, são caracterizados pela dependência ou heterogeneidade espacial. Neste caso, infelizmente, os resultados obtidos pela metodologia tradicional não são capazes de explicar com fidedignidade o comportamento do mercado habitacional, uma vez que podem apresentar problemas de tendenciosidade, inconsistência ou ineficiência. A solução é proceder à análise com base nos modelos espaciais, isto é, modelos estimados pela metodologia denominada Econometria Espacial.

O presente trabalho pretende atingir dois objetivos: um relacionado aos aspectos metodológicos e outro de natureza empírica. O de caráter metodológico consiste em mostrar como se pode diagnosticar e incorporar os efeitos espaciais na especificação

3 Desenvolvido por Dipasquale e Wheaton (1994).

4 No Brasil, dispõe-se apenas de dados da PNAD, contudo são dados incompletos que não permitem uma estimativa segura da Função de Demanda Habitacional.

5 No restante no texto, essa abordagem será denominada de Econometria Tradicional. Tal denominação é utilizada para enfatizar a distinção entre a mesma e a abordagem espacial que será utilizada adiante. 
de modelos que visam explicar o comportamento do mercado habitacional, isto é, modelos de preços hedônicos e de demanda por habitação, utilizando-se uma metodologia apresentada pela econometria espacial. O segundo trata da pesquisa aplicada ao estudo da economia urbana, isto é, a análise microeconômica do mercado habitacional, onde será estimada uma função demanda por habitação para o Recife, capital do Estado de Pernambuco, uma das mais importantes cidades do País. ${ }^{6}$

A função de demanda habitacional será estimada em dois estágios, adotando-se os procedimentos utilizados por Ermisch et al. (1996) e Tiware et al. (1999), incorporando-se aos mesmos os efeitos espaciais, por meio da modelagem por econometria espacial. Isto é, no primeiro estágio, será estimado um modelo espacial de preços hedônicos para a cidade do Recife, pelo qual será calculado o Índice de Preço da Habitação (IPH). A razão entre a despesa com a habitação, representada pelo seu preço de compra e o IPH correspondente ao bairro e ao período em que a mesma foi demandada, será usada como medida de consumo de serviços de habitação. Por fim, será estimado um modelo espacial de demanda habitacional, através de uma regressão do consumo de serviços de habitação sobre os preços relativos da habitação (IPH), a renda familiar e outras variáveis que afetam a demanda, relativas à família e embasadas na operação de aquisição da habitação.

Para realização dos testes empíricos e estimação dos modelos espaciais, serão utilizados dados do Censo Demográfico do IBGE (2000) e de habitações financiadas pela CAIXA. A partir dos resultados obtidos pelo modelo espacial de demanda habitacional, será possível estimar a elasticidade-renda e elasticidade-preço da habitação, bem como o comportamento da demanda em relação a sexo, idade, nível de poupança do chefe da família e prazo de financiamento, que são informações fundamentais para formulação e implantação de políticas habitacionais.

Este trabalho está organizado em quatro seções, além desta introdução. Na seção 2 , apresenta-se a base teórica da estimação empírica que será realizada nas seções seguintes. Discute-se a metodologia tradicional de estimação de preços hedônicos e da demanda habitacional, mostra-se como diagnosticar a presença de efeitos espaciais e como incorporar estes efeitos aos modelos, ou seja, como utilizar a técnica de modelagem por econometria espacial. A seção 3 ocupa-se da estimação de um modelo espacial de preços hedônicos, utilizando-se o banco de dados da CAIXA, referente a habitações demandadas por meio da instituição. Com base neste modelo, analisa-se o comportamento do consumidor ante as características da cesta de habitação com que se defronta no mercado e constrói-se um Índice de Preços da Habitação. Esta seção ainda apresenta um modelo espacial de demanda por habitação, utilizando-se o Índice de Preços da Habitação, juntamente com a renda familiar

6 Recife é a $7^{\mathrm{a}}$ capital do País em população e a $8^{\mathrm{a}}$ em número de domicílios. 
e outras características sociodemográficas da família e da operação de aquisição da habitação, que possam afetar a demanda. Finalmente, na seção 4, são apresentadas as conclusões.

\section{A Modelagem Tradicional da Equação de Preços Hedônicos e a Econome- tria Espacial}

A formulação mais simples da equação de preços hedônicos, relacionada com o comportamento do mercado habitacional, é representada por:

$$
Y=X \beta+\varepsilon
$$

sendo $Y$ um vetor de preços dos imóveis, $X$ as variáveis explicativas, $\beta$ o vetor de parâmetros a estimar e $\varepsilon$ o vetor de erros aleatórios do modelo de regressão, respectivamente.

Tradicionalmente, esta estimação tem sido realizada pelo método dos mínimos quadrados ordinários (MQO). O vetor de coeficientes do modelo, também chamado de vetor de preços implícitos ou hedônicos das características da habitação, é obtido pela equação (2):

$$
\hat{\beta}=\left(X^{\prime} X\right)^{-1}\left(X^{\prime} Y\right)
$$

O coeficiente $b j$, de uma característica $X j$, corresponde ao preço hedônico de uma unidade desta característica. Desta forma, o valor médio estimado para um imóvel particular, de características representadas por um vetor $\mathrm{C}=\left[\begin{array}{llll}1 & c 1 & c 2 \ldots & c k\end{array}\right]$, com base num modelo com $\mathrm{k}$ variá,veis independentes e vetor de parâmetros estimados dado por $\hat{\beta}=\left[\beta_{0} \beta_{1} \beta_{2} \ldots \beta_{k}\right]$, é calculado pela expressão:

$$
Y c=C \hat{\beta}
$$

Constata-se que, da combinação linear dos preços implícitos de cada uma das características da habitação e as respectivas quantidades demandadas, chega-se ao valor estimado de mercado da habitação. A norma brasileira para avaliação de imóveis urbanos, NBR-14.653-2:2004, da Associação Brasileira de Normas Técnicas ABNT, denomina este procedimento avaliatório de Método Comparativo de Dados de Mercado. 
$\mathrm{Na}$ abordagem econométrica acima citada, a questão espacial, quando considerada, é tratada tomando-se como referência distâncias a polos de influência ou setorização das cidades, admitindo-se a independência espacial da variável explicada e dos resíduos do modelo de regressão. Contudo, segundo Anselin (1988), dados associados à posição que ocupam no espaço (cidades, regiões, bairros ou a própria coordenada geográfica), como é o caso da habitação, podem conter erros de medidas em relação à exata localização do imóvel, como também efeitos de interação, difusão ou "spillovers" espaciais. Esses efeitos acabam por ser traduzir em dependência ou em heterogeneidade espacial.

A autocorrelação ou dependência espacial pode afetar o termo de erro, a variável dependente ou ambos. A não consideração deste efeito, como vem ocorrendo rotineiramente na análise do comportamento do mercado habitacional, pode gerar sérios problemas de estimação, pois, na presença de autocorrelação espacial nos resíduos, os parâmetros estimados por (2) são ineficientes e, neste caso, testes de significância como t de Student e F de Snedecor, bem como os intervalos de confiança inferidos, não são mais válidos, e as decisões tomadas com base neles são enganosas. Da mesma forma, a dependência espacial dos preços observados em relação aos preços dos imóveis vizinhos provocará estimações tendenciosas e inconsistentes para os parâmetros, em virtude de um erro de especificação no modelo, pela não inclusão de uma variável dependente espacialmente defasada no modelo (1). Em ambos os casos, a estimação por MQO mostra-se inadequada, devendo ser substituído por modelos espaciais.

O primeiro passo na utilização desta metodologia é o diagnóstico da presença de efeitos espaciais. A dependência ou autocorrelação espacial ${ }^{7}$ tem sido diagnosticada na literatura de duas formas distintas: pela análise gráfica do variograma ou utilizando-se testes estatísticos específicos, que utilizam matrizes de vizinhança. No primeiro caso, a inferência espacial é realizada pelo processo denominado de Krigeagem, ${ }^{8}$ que tem como fundamento a Teoria das Variáveis Regionalizadas (TVR), desenvolvida por Matheron (1965); no segundo caso, a modelagem espacial é realizada conforme a metodologia desenvolvida por Anselin (1988), que será utilizada neste artigo. ${ }^{9}$

7 Além da autocorrelação ou dependência espacial, existe também um outro efeito que pode ser encontrado nos dados distribuídos espacialmente: a heterogeneidade espacial, que diz respeito à instabilidade dos parâmetros em relação à região em que se situam os dados e, na ausência de dependência espacial, pode ser diagnosticado pelo teste de Chow e tratado pela metodologia tradicional, utilizando-se variáveis de interação entre cada variável explicativa e variável indicativa da região (ver DANTAS, 1997 e DANTAS; ROCHA, 2002).

8 Denominação dada por George Matheron em homenagem ao estatístico sulafricano, Daniel Gerhardus Krige, que, em 1951, desenvolveu as bases para os problemas de estimação espacial (OLMO,1994).

9 Uma boa resenha sobre a metodologia de Krigeagem pode ser encontrada em Burrough e McDonnell (1998) ou Olmo (1994). 


\subsection{Testes de Dependência Espacial}

Para diagnosticar a presença de efeitos de dependência espacial, bem como introduzir estes efeitos no modelo (1), pela metodologia desenvolvida por Anselin (1988), é necessário definir, previamente, uma matriz de pesos espaciais, conhecida como $W$. No caso mais simples, $W$ é uma matriz simétrica em que cada elemento $w_{i j}$, é igual a $1(\mathrm{um})$, se $i$ e $j$ são vizinhos, e igual a zero, no caso contrário. Por convenção, os elementos diagonais são iguais a zero, ou seja, $w_{i i}=0$. Outras matrizes, como as propostas por Cliff e Ord (1981) consideram a importância dos vizinhos por meio de uma ponderação correspondente ao inverso da distância ou ao inverso do quadrado da distância entre eles.

Em geral, a matriz de $W$ é padronizada por linha, assumindo a nomenclatura $W s$. Neste caso, cada elemento de $W_{s}$, representado por $w_{i j}^{s}$, é obtido dividindo-se $w_{i j}$ pela soma dos elementos da linha $i$ a que pertence, ou seja $w_{i j}^{s}=w_{i j} / \sum_{j} w_{i j}$. Nesta matriz, os elementos das linhas somam l. Este procedimento, além de facilitar a interpretação dos pesos, como uma média ponderada dos valores dos vizinhos, assegura a compatibilidade entre os modelos (ANSELIN; BERA, 1998).

Os principais testes utilizados para detectar a autocorrelação espacial são Moran I, LM $^{10}$ Robusto (erro) e LM Robusto (defasagem). O teste de Moran I é o mais usado nos estudos de dados de corte transversal de unidades geográficas. O problema deste teste é que ele não identifica o tipo de efeito (erro ou defasagem espacial). Por isso, serão utilizados testes mais específicos: o LM (erro) Robusto, para detectar efeitos de autocorrelação espacial no termo de erro, e o LM (defasagem) Robusto, para verificar a presença de efeitos de defasagem espacial na variável dependente. Maiores detalhes podem ser encontrados em Anselin (1988a). É importante frisar que a validade destes testes exige a aceitação das hipóteses de normalidade e homocedasticidade dos resíduos de MQO, obtidos pelo modelo (1).

\subsection{Os Modelos Espaciais}

Uma vez detectada a presença de autocorrelação espacial nos dados, faz-se necessário introduzir extensões convenientes no modelo tradicional, representado na equação (1), considerando-se os efeitos autocorrelação espacial nos erros, através do Modelo de Erro Espacial ou os efeitos ocasionados pelas interações entre os valores da variável dependente, pelo Modelo de Defasagem Espacial, como mostra-se a seguir.

10 Sigla de Lagrange Multiplier. 


\subsubsection{O Modelo de Erro Espacial}

A autocorrelação espacial no termo de erro está relacionada a erros de medida ocasionados pelas divisões artificiais das unidades geográficas, como os limites estabelecidos para os bairros de uma cidade, que não necessariamente coincidem com a verdadeira dimensão do fenômeno observado. Isto é, na prática, o consumidor não tem o conhecimento exato dos limites que dividem os bairros. No mercado habitacional, há uma tendência de efeito de transbordamento de um bairro de maior importância sobre os seus vizinhos. Por exemplo, o bairro de Boa Viagem, no Recife, devido à sua importância no contexto urbano e à grande demanda por habitação, foi se estendendo sobre os bairros do Pina e Imbiribeira. Outro fator que pode gerar a autocorrelação espacial nos erros é a omissão de variáveis locacionais relevantes.

Para tratar adequadamente este tipo de efeito espacial nos dados, a primeira modificação com relação à equação (1) será considerar o processo espacial autoregressivo no termo de erro, da seguinte forma:

$$
\varepsilon=\lambda W \varepsilon+u \quad \text { ou } \quad \varepsilon=(I-\lambda W)^{-1} u
$$

onde $\lambda$ representa o coeficiente de autocorrelação espacial do termo de erro; $u$ é normalmente distribuído com média zero e variância constante; $I$ é a matriz identidade e $W$ a matriz de pesos espaciais ponderada. Substituindo (4) em (1) resulta no seguinte modelo de erro espacial:

$$
Y=X \beta+(I-\lambda W)^{-1} u
$$

Para obtenção de estimativas eficientes dos parâmetros do modelo (8), é necessário usar o estimador de verossimilhança, que consiste em maximizar a função de logverossimilhança dada por (6).

$$
L=\frac{n}{2} \ln (\pi)-\frac{n}{2} \ln \left(\sigma^{2}\right)+\ln |\mathbf{I}-\lambda \mathbf{W}|-\frac{1}{2 \sigma^{2}} \varepsilon^{\prime}(\mathbf{I}-\lambda \mathbf{W})^{\prime}(\mathbf{I}-\lambda \mathbf{W}) \varepsilon
$$

onde $n$ representa o número de dados da amostra, $l n$ o símbolo do logaritmo natural, $\sigma^{2}$ a variância do modelo e as demais variáveis têm a mesma definição da equação (4).

Como já observado quando os erros são autocorrelacionados espacialmente, os parâmetros estimados pelo modelo tradicional (1) são não eficientes, isto é, os desvios padrão que se encontram associados a eles são tendenciosos. 


\subsubsection{O Modelo de Defasagem Espacial}

O efeito de defasagem espacial é ocasionado pela dependência espacial criada através de uma interação espacial entre os preços dos imóveis, uma espécie de "efeito de vizinhança” na determinação dos preços. Neste caso, esta influência é medida pela inclusão de uma variável adicional no modelo (1), dada por $W Y$, que é a variável dependente espacialmente defasada. Cada elemento $w y_{i}$, do vetor $W Y$, é formado por uma ponderação dos preços dos imóveis vizinhos. Esta variável serve também para captar os efeitos de dependência espacial não considerados explicitamente nas variáveis locacionais comumente utilizadas, como questões ligadas à segurança, saúde, tráfego, educação, etc. (PACE; BARRY; SIRMAMS, 1998). Com a incorporação desta variável, o modelo (1) passa a ser

$$
Y=X \beta+\rho W Y+\varepsilon
$$

onde $\rho$ é o coeficiente de autocorrelação espacial da variável $W Y$ e $\varepsilon$ é idêntica e independentemente distribuído (i.i.d).

Observe-se que, ao comparar os modelos (1) com (7), constata-se no primeiro a falta da variável explicativa $\boldsymbol{W} \boldsymbol{Y}$, o que gera um grave erro de especificação. Neste caso, as avaliações realizadas por (1) são tendenciosas e inconsistentes. Da mesma forma que no modelo (5), a estimação deve ser realizada pelo método da máxima verossimilhança, que consiste na maximização da função (8), ${ }^{11}$ utilizando técnicas de otimização não linear.

$$
L=\frac{n}{2} \ln (\pi)-\frac{n}{2} \ln \left(\sigma^{2}\right)+\ln |\mathbf{I}-\rho \mathbf{W}|-\frac{1}{2 \sigma^{2}} \varepsilon^{\prime} \varepsilon
$$

\subsubsection{Escolha de Modelos}

Uma maneira de escolher o modelo a adotar - o modelo de erro espacial ou o modelo de defasagem espacial - pode ser feita pela comparação do valor absoluto das estatísticas (5) e (6). Assim, quanto maior for o valor encontrado na estatística de teste, maior será o efeito espacial correspondente a esta estatística, conforme argumento de Anselin e Rey (1991). Outra forma de escolha de modelos é pela utilização dos critérios de informação de Akaike (AIC) e de Schwartz (SC). Na escolha de modelos alternativos, o melhor será aquele com menores valores encontrados para AIC e SC. Uma discussão mais detalhada destes critérios de informação pode ser encontrada em Akaike (1981) e Anselin (1988a).

11 Uma derivação de estimador de verossimilhança pode ser encontada em Anselin (1988). 


\section{Resultados Empíricos}

\subsection{Modelo Espacial de Preços Hedônicos}

Esta seção trata da estimação empírica de um modelo espacial de preços hedônicos para a cidade do Recife, que representa o primeiro passo no tratamento da estimação da função de demanda habitacional, objetivo principal deste trabalho. Será utilizada uma base de dados da CAIXA, referente aos imóveis financiados pela instituição, no período de junho de 2000 a junho de 2002. No banco de dados pesquisado, verificou-se que $88 \%$ da demanda por unidades habitacionais é por apartamentos, razão pela qual optou-se por analisar a demanda por esta tipologia. Do banco de dados, foram extraídas as informações sobre as características locacionais (logradouro, número e bairro) e estruturais dos apartamentos (área privativa, idade, conservação, número de quartos sociais, suítes, vagas de estacionamento, pavimentos do edifício, elevadores e de unidades da edificação), preço e data da compra, obtendo-se uma amostra de 232 dados, distribuídos em 36 bairros da cidade, que representa $21,20 \%$ dos apartamentos demandados no período.

Inicialmente, será analisado o modelo tradicional de preços hedônicos e, com base nos resíduos deste modelo, será realizado o diagnóstico da presença de efeitos de dependência espacial. Sendo o resultado positivo, o modelo espacial de preços hedônicos será estimado com base na metodologia de econometria espacial. ${ }^{12}$

O modelo tradicional de preços hedônicos, o preço da habitação (P) é função das suas características estruturais (E), locacionais (L) e ainda da época em que a mesma foi demandada (T), como definido na equação (9).

$$
P=f(E, L, T, \beta)+\varepsilon
$$

onde $f$ é um operador indicativo da forma funcional, $\beta$ são parâmetros e $\varepsilon$ os erros aleatórios do modelo, respectivamente. Considera-se $\varepsilon$ como iid.

A estimação do modelo (9) é realizada via $\mathrm{MQO}$, na forma funcional semi-log. Isto é, faz-se uma regressão do logaritmo neperiano do preço de compra da habitação (P) sobre as variáveis explicativas correspondentes ao nível socioeconômico do bairro onde foi demandada (Renda do Bairro); número de quartos sociais do apartamento, de suítes e vagas na garagem; número de pavimentos, dummy para existência de elevadores; idade e conservação do edifício; período em que foi demandado o imóvel

12 As regressões foram rodadas no programa SpaceStat, desenvolvido por Anselin (1990). 
e distâncias do edifício à Praia de Boa Viagem, ao Centro de Negócios da Cidade e ao Parque da Jaqueira.

Os resultados para o modelo de preços hedônicos é apresentado na Tabela $1 .{ }^{13}$ Podese observar que os sinais obtidos para os coeficientes das variáveis independentes estão coerentes com o mercado, pois há expectativas de elevação dos preços dos apartamentos com aumento do padrão socioeconômico do bairro onde está localizado, com a quantidade de metros quadrados de área privativa, de quartos sociais, de suítes e de vagas na garagem. É esperado, também, que as unidades situadas em edifícios com maior número de pavimentos, de elevadores e de melhor conservação, sejam mais valorizadas e, ainda, que os apartamentos localizados em edifícios mais velhos e com grande número de unidades sejam mais baratos. Em relação aos polos de influência, verifica-se que os apartamentos são mais valorizados à medida que se aproximam da Praia de Boa Viagem e também do Parque da Jaqueira, comprovando a importância destes dois polos de influência no contexto urbano, mas se desvalorizam ao se aproximarem do Centro de Negócios da Cidade, o que denota indícios de uma estrutura multicêntrica para a cidade do Recife. Os coeficientes da grande maioria das variáveis explicativas mostraram-se estatisticamente significantes ao nível de $1 \%$, com exceção das variáveis SU, VA e PE. O modelo apresenta um alto poder explicativo, com coeficiente de determinação de 0,90 , e a hipótese nula de que o conjunto de variáveis explicativas adotadas não é importante para explicar a variabilidade observada nos preços dos apartamentos é fortemente rejeitada quando utilizado o teste $\mathrm{F}$.

Apesar dos bons resultados estatísticos obtidos na estimação, deve-se notar que uma importante questão ainda não foi levada em consideração: a dimensão espacial. A rigor, nada se pode concluir a respeito dos parâmetros deste modelo, antes de testar a autocorrelação espacial.

13 Quatro dados foram excluídos durante a análise utilizando-se o critério da distância de Cook (1977). 
Tabela 1 - Modelo Tradicional de Preços Hedônicos para o Recife (Forma Funcional Semi-log)

\begin{tabular}{|c|c|c|c|c|}
\hline Variável & Coeficiente & Desvio Padrão & Estatística t & Probabilidade \\
\hline Constante & 10,152 & 0,127 & 79,658 & 0,000 \\
\hline Renda do Bairro & 0,012 & 0,004 & 3,244 & 0,001 \\
\hline Área Privativa & 0,006 & 0,001 & 9,050 & 0,000 \\
\hline Quartos Sociais & 0,072 & 0,029 & 2,452 & 0,015 \\
\hline Número de Suítes & 0,036 & 0,021 & 1,686 & 0,093 \\
\hline Número de Vagas na Gararem & 0,047 & 0,034 & 1,397 & 0,164 \\
\hline Número de Pavimentos & 0,021 & 0,004 & 4,833 & 0,000 \\
\hline Elevadores & 0,100 & 0,027 & 3,640 & 0,000 \\
\hline Número de Unidades & $-0,003$ & 0,001 & $-2,957$ & 0,003 \\
\hline Idade & $-0,010$ & 0,002 & $-4,769$ & 0,000 \\
\hline Conservação & 0,071 & 0,019 & 3,644 & 0,000 \\
\hline Período da Compra & 0,048 & 0,029 & 1,687 & 0,093 \\
\hline Distância Praia & $-0,066$ & 0,017 & $-3,819$ & 0,000 \\
\hline Distância Centro & 0,082 & 0,023 & 3,627 & 0,000 \\
\hline Distância Jaqueira & $-0,077$ & 0,020 & $-3,884$ & 0,000 \\
\hline $\mathrm{R}^{2}$ & 0,904 & & & \\
\hline $\mathrm{R}^{2}$-adj & 0,898 & & & \\
\hline AIC & $-97,616$ & & & \\
\hline SC & $-46,176$ & & & \\
\hline F-Teste ( Probabilidade) & 144,77 & $(0,00)$ & & \\
\hline \multicolumn{5}{|l|}{ Teste de normalidade } \\
\hline Jarque-Bera (prob) & 0,61 & $(0,74)$ & & \\
\hline \multicolumn{5}{|l|}{ Heterocedasticidade Breusch- } \\
\hline Pagan (prob) & 19,91 & $(0,13)$ & & \\
\hline
\end{tabular}

Notas: AIC significa critério de informação de Akaike e CS é o critério de Scharwz.

O diagnóstico da presença de efeitos de dependência espacial foi realizado utilizando-se os testes LM Robusto (erro) e LM Robusto (defasagem) sobre os resíduos de MQO do modelo estimado na Tabela 1 . Trabalhou-se com uma matriz de vizinhança $W$, seguindo a forma proposta por Cliff e Ord (1981), onde cada elemento da matriz, $w_{i j}$, representa o inverso da distância entre os edifícios $i$ e $j$, medida em hectômetros $(\mathrm{hm})$. A Tabela 2 apresenta os resultados para os testes de dependência espacial. O teste LM Robusto (defasagem) indica um forte efeito de interação espacial nos preços dos apartamentos, uma vez que se mostrou significante a menos de $2 \%$. Ou seja, o teste indica que o efeito vizinhança parece afetar os preços dos apartamentos. 
Tabela 2 - Diagnóstico da Dependência Espacial para o Recife

\begin{tabular}{lccc}
\hline Teste & MI/GL & Valor & Probabilidade \\
\hline LM Robusto (erro) & 1 & 0,469 & 0,493 \\
LM Robusto (defasagem) & 1 & 5,508 & 0,019 \\
\hline
\end{tabular}

Diante dessa possibilidade de dependência espacial nos preços observados ou efeito vizinhança, estima-se o modelo de defasagem espacial de preços hedônicos, seguindo os procedimentos apresentados acima.

O modelo de defasagem espacial de preços hedônicos, na forma funcional semilog, é uma expansão do modelo (9), pela inclusão da variável dependente defasada espacialmente, $W \_\ln P$, conforme equação (10).

$$
\ln P=f\left(\boldsymbol{W}_{-} \ln P, E, L, T, \rho, \beta\right)+\varepsilon
$$

onde $l n$ é a sigla dos logaritmos neperianos, $W$ a matriz de vizinhança definida na seção anterior, $\rho$ o parâmetro correspondente à $W \_l n P$, conhecido como coeficiente de autocorrelação espacial, e as demais variáveis têm a mesma descrição do modelo (9). Cada elemento da variável $W_{-} \ln P$, ou seja $w_{-} \ln p_{i}$, representa a média ponderada dos logaritmos dos preços dos apartamentos vizinhos ao apartamento $i$, onde o peso de cada vizinho $j$ é ponderado pelo inverso da distância entre os apartamentos $i$ e $j$. Desta forma, a influência das negociações de apartamentos realizadas sobre um determinado vizinho, é tanto maior quanto menor for a distância entre eles e o dado apartamento.

Os resultados da estimação espacial, apresentados na Tabela 3, indicam que o coeficiente $\rho$, da variável defasada espacialmente, $W \ln P$, é positivo e estatisticamente significante a menos de $1 \%$, implicando a existência de um forte efeito de defasagem espacial positiva na amostra. Neste caso específico, a formação dos preços de mercado da habitação não é explicada apenas pelas suas características estruturais, locacionais e temporais, tradicionalmente adotadas, mas também depende dos preços dos apartamentos vizinhos, isto é, o preço de compra é influenciado positivamente pelos preços dos apartamentos vizinhos. Pode-se observar que houve modificações significativas nos resultados das estimações dos parâmetros e respectivos desvios padrão, quando comparados com os apresentados na Tabela 3.5 (modelo tradicional), fato não surpreendente, uma vez que o efeito de defasagem espacial é significante e a exclusão da variável espacialmente defasada no modelo tradicional gera tendenciosidades e ineficiências nas estimativas. Verifica-se que os coeficientes das variáveis tiveram seus desvios padrão reduzidos, notadamente nas variáveis Vagas na Garagem e Período da Compra, que sofreram alterações de 16,37\% para 
$10,02 \%$ e $9,30 \%$ para $5,27 \%$, respectivamente, nas probabilidades de se cometer um erro do tipo I. Observa-se, também, que as alterações mais significativas nas magnitudes dos coeficientes ocorreram no intercepto e nas variáveis relacionadas à localização, que tiveram seus valores reduzidos. Estas mudanças podem ser atribuídas ao erro de especificação no modelo tradicional, pela não inclusão da variável $W \_\ln P$, que contribuiu para gerar tendenciosidades sérias nos seus coeficientes. Isto ocorre porque o modelo tradicional, ao desconsiderar o efeito vizinhança, supõe que cada apartamento tem a mesma influência sobre os demais, independentemente da distância entre eles e grande parte da variabilidade espacial é absorvida pela média geral, representada pelo intercepto.

Tabela 3 - Resultados do Modelo de Defasagem Espacial para o Recife

\begin{tabular}{lrccc}
\hline Variável & Coeficiente & Desvio Padrão & Estatística $z$ & Probabilidade \\
\hline$W_{-}$lnP & 0,2061 & 0,0741 & 2,7796 & 0,0054 \\
Constante & 7,8926 & 0,8267 & 9,5473 & 0,0000 \\
Renda do Bairro & 0,0072 & 0,0039 & 1,8445 & 0,0651 \\
Área Privativa & 0,0059 & 0,0006 & 9,4066 & 0,0000 \\
Quartos Sociais & 0,0648 & 0,0280 & 2,3150 & 0,0206 \\
Número de Suítes & 0,0333 & 0,0201 & 1,6517 & 0,0986 \\
Número de Vagas na Gararem & 0,0531 & 0,0323 & 1,6440 & 0,1002 \\
Número de Pavimentos & 0,0215 & 0,0041 & 5,2095 & 0,0000 \\
Elevadores & 0,0925 & 0,0262 & 3,5372 & 0,0004 \\
Número de Unidades & $-0,0030$ & 0,0010 & $-3,0031$ & 0,0027 \\
Idade & $-0,0097$ & 0,0019 & $-5,0422$ & 0,0000 \\
Conservação & $-0,0687$ & 0,0185 & $-3,7140$ & 0,0002 \\
Período da Compra & 0,0528 & 0,0273 & 1,9373 & 0,0527 \\
Distância Praia & $-0,0415$ & 0,0187 & $-2,2233$ & 0,0262 \\
Distância Centro & 0,0615 & 0,0227 & 2,7034 & 0,0069 \\
Distância Jaqueira & $-0,0587$ & 0,0202 & $-2,8998$ & 0,0037 \\
$R^{2}$ & 0,91 & & & \\
AlC & $-102,70$ & & & \\
SC & $-47,83$ & & & \\
\hline
\end{tabular}

Notas: AIC significa critério de informação de Akaike e CS é o critério de Scharwz.

Outro aspecto importante é que, utilizando-se os critérios da informação de Akaike (AIC) e Schwartz (SC), os valores obtidos para o modelo espacial são inferiores aos encontrados no modelo tradicional, como mostrado na Tabela 4, o que mostra a melhoria do ajustamento pela metodologia de econometria espacial. 
Tabela 4 - Comparação entre o Modelo Tradicional (MT) e o Modelo Espacial (ME)

\begin{tabular}{lcc}
\hline Critério & MT & ME \\
\hline AIC & $-97,61$ & $-102,70$ \\
SC & $-46,18$ & $-47,83$ \\
\hline
\end{tabular}

Notas: AIC significa critério de informação de Akaike e CS é o critério de Scharwz.

\subsection{O Modelo Espacial de Demanda Habitacional}

Esta seção é dedicada à estimação empírica de um modelo espacial de demanda por habitação para a cidade do Recife, seguindo o processo de estimação em dois estágios utilizado por Ermisch et al. (1996) e Tiware et al. (1999), com incorporação da componente espacial. No primeiro estágio, utiliza-se o modelo espacial de preços hedônicos para construção de um Índice de Preços da Habitação (IPH), correspondente ao valor de mercado estimado pelo referido modelo, para um apartamento típico, com as condições medianas dos dados amostrais. Ou seja, um apartamento com 71,28 $\mathrm{m}^{2}$ de área privativa, dois quartos sociais, sem suíte, com uma vaga na garagem, situado em um prédio do tipo caixão ${ }^{14}$ com 16 unidades, 12 anos de idade e conservação regular. O IPH é calculado considerando-se a localização variável para cada bairro abrangido pela pesquisa e também para cada período em que a unidade foi demandada. A razão entre o preço de compra e o IPH correspondente é definido como o consumo de habitação $(\mathrm{CH})$, que representará a variável dependente na equação de demanda por habitação. No segundo estágio, a equação de demanda habitacional será estimada pela regressão de $\mathrm{CH}$ sobre o preço relativo da habitação (IPH), a renda e outras variáveis sociodemográficas da família e da operação de aquisição, que possam afetar a demanda.

A base de dados utilizada refere-se a apartamentos financiados pela CAIXA, no período de junho/2000 a junho/2002. Com uma amostra de 228 dados, realizou-se a estimação empírica de um modelo espacial de preços hedônicos, que representou o primeiro passo para estimação da função de demanda por habitação. Para complementação da base de dados necessária à conclusão da estimação desta função, foram extraídas as seguintes informações disponíveis: renda familiar, sexo e idade do mutuário, prazo de financiamento e o sinal ou parte utilizada de recursos próprios na compra do apartamento, denominada de poupança. Tendo em vista que alguns dos dados iniciais referem-se a apartamentos adquiridos totalmente com recursos de FGTS do demandante, a declaração da renda não foi exigida pela CAIXA, o que gerou a perda de 34 informações, restando uma amostra de 194 dados.

14 Denominação dada a edificações de até quatro pavimentos, sem elevador. 
Um resumo da amostra utilizada na estimação empírica com indicação das variáveis utilizadas e suas siglas adotadas, valores mínimo, médio, mediano e máximo, bem como a amplitude, encontra-se na Tabela 5.

Tabela 5 - Descrição da Amostra Utilizada para Estimação da Função de Demanda por Habitação na Cidade do Recife

\begin{tabular}{lcrrrrr}
\hline Variável & Sigla & Mínimo & Média & Mediana & Máximo & Amplitude \\
\hline Sexo ${ }^{(1)}$ & SX & 1,00 & 1,44 & 1,00 & 2,00 & 1,00 \\
Idade & $\mathrm{ID}$ & 22,00 & 38,24 & 37,00 & 69,00 & 47,00 \\
Renda & $\mathrm{RE}$ & 476,30 & $2.286,43$ & $1.600,00$ & $17.000,00$ & $16.523,70$ \\
Prazo Amortização & $\mathrm{PZ}$ & 1,00 & 178,79 & 240,00 & 300,00 & 299,00 \\
Poupança & $\mathrm{PO}$ & 95,58 & $22.048,57$ & $13.493,99$ & $183.505,50$ & $183.409,90$ \\
Índice de Preço & $\mathrm{IPH}$ & $24.587,66$ & $30.790,50$ & $30.031,44$ & $42.192,59$ & $17.604,93$ \\
Consumo Habitação & $\mathrm{CH}$ & 0,56 & 1,44 & 1,13 & 8,76 & 8,20 \\
\hline
\end{tabular}

Nota: (1) Adotou-se valor 1 para o sexo masculino e 2 para o feminino.

Pelos resultados da Tabela 5, pode-se observar a grande heterogeneidade dos dados: as idades variam de 22 a 69 anos, atingindo uma idade mediana de 37 anos; a renda familiar mínima é de $\mathrm{R} \$ 476,30$, enquanto que a máxima é de $\mathrm{R} \$ 17.000,00$, que corresponde a 40 vezes a menor renda, sendo a renda familiar mediana de $\mathrm{R} \$$ 1.600,00; o prazo de amortização varia de 1 a 300 meses, ${ }^{15}$ atingindo um valor mediano de 240 meses; a parte de recursos próprios utilizada ou recursos do FGTS varia de $R \$ 95,58$ a $R \$ 183.505,50$, com valor mediano de $R \$ 13.493,99$. Verificase que um apartamento com as mesmas características estruturais varia de $\mathrm{R} \$$ $24.587,66$ até $\mathrm{R} \$ 42.192,59$, dependendo da sua localização na cidade, indicando que a localização pode representar quase $72 \%$ do valor do imóvel. A amplitude do consumo de habitação também é significativa: varia de 0,56 a 8,76. Isto é, o maior consumo de habitação é quase 16 vezes superior que o menor consumo. Apenas em relação ao sexo, existe equilíbrio entre mutuários, apresentando uma pequena prevalência pelo sexo feminino.

Com base nesta amostra, será ajustado o modelo tradicional de demanda habitacional e os seus resíduos serão utilizados no diagnóstico da presença de efeitos de dependência espacial. Sendo o resultado positivo, o modelo espacial de demanda habitacional será estimado pela metodologia de Econometria Espacial, como segue.

15 O prazo de amortização de 1 mês se refere à compra com utilização total do FGTS 
O ajustamento do modelo tradicional de demanda habitacional assume, geralmente, a forma da equação (11).

$$
C H=f(I P H, R E, S, \beta)+\varepsilon
$$

onde $\mathrm{CH}$ é o vetor de consumo de habitação; IPH é o vetor de preço relativo da habitação ou Índice de Preço da Habitação estimado para cada região, em cada período; $R E$, o vetor correspondente à renda familiar; $S$, o vetor de outras características sociodemográficas que podem afetar a demanda, tais como: sexo, idade, nível de riqueza, tamanho da família, nível de ocupação e instrução do chefe da família e do cônjuge, bem como outras riquezas humanas e não humanas, e $\beta$ e $\varepsilon$ são os vetores de parâmetros e dos erros aleatórios do modelo, respectivamente. Para o caso em pauta, como se trata de imóveis financiados, inclui-se também, no modelo, o prazo de financiamento. Considera-se $\varepsilon$ como iid, ou seja, que os erros aleatórios são idêntica e independentemente distribuídos (normais, homocedásticos e não autocorrelacionados).

Uma questão importante a ressaltar é que, neste estudo, não se dispõe de informações relativas ao nível de ocupação e de instrução do chefe da família ou do cônjuge, nem sobre o tamanho da família. Estes dados estão em outro sistema da CAIXA: o SIRIC - Sistema de Risco de Crédito, não disponibilizado para consulta. Desta forma não foi possível estimar a renda permanente, o que pode gerar subestimação da elasticidade-renda. Como visto na seção anterior, dispõe-se apenas das seguintes variáveis adicionais, que podem afetar a demanda por habitação: sexo e idade do cabeça do casal, prazo de financiamento e poupança utilizada na compra do apartamento. Assim, com a inclusão da variável idade será possível a análise do padrão de consumo do indivíduo durante o ciclo de vida, e a poupança utilizada na compra funciona como uma proxy para o nível de riqueza da família.

Para estimação empírica do modelo tradicional de demanda por habitação na cidade do Recife, definido na equação (11), foi utilizado o modelo clássico de regressão, tomando-se por base as estimações via MQO, cujos resultados encontram-se na Tabela 6. Isto é, foi feita uma regressão do consumo de habitação $(\mathrm{CH})$ sobre as seguintes variáveis independentes: índice de preços da habitação (IPH), renda familiar (RE), sexo (SX), idade (ID), prazo de financiamento (PZ) e poupança (PO). $\mathrm{O}$ ajustamento foi realizado na forma tradicional log-linear, ou de elasticidade constante, que tem sido a forma mais utilizada na literatura, flexibilizando apenas as variáveis sexo e idade, que foram tratadas na escala original. Para captar as variações de consumo durante o ciclo de vida do indivíduo, introduziu-se também a variável idade elevada ao quadrado $\left(\mathrm{ID}^{2}\right)$, uma vez que é comum a existência de um ponto de máximo consumo durante este período. 
Tabela 6 - Ajustamento do Modelo Tradicional de Demanda Habitacional para o Recife

\begin{tabular}{lcccc}
\hline Variável & Coeficiente & Desvio Padrão & Estatística t & Probabilidade \\
\hline Constante & $-3,01748$ & 1,68639 & $-1,78931$ & 0,07519 \\
InRE & 0,55222 & 0,03150 & 17,52698 & 0,00000 \\
InIPH & $-0,23541$ & 0,17364 & $-1,35572$ & 0,17683 \\
SX & 0,08156 & 0,03657 & 2,23024 & 0,02692 \\
ID & $-0,00323$ & 0,00200 & $-1,61301$ & 0,10843 \\
ID & $4,24 \mathrm{E}-05$ & 0,00015 & 0,27133 & 0,78643 \\
InPZ & 0,06488 & 0,01138 & 5,69745 & 0,00000 \\
InPO & 0,12180 & 0,01291 & 9,43594 & 0,00000 \\
$R^{2}$ & 0,7535 & & & \\
$R^{2}$ - Ajustado & 0,7442 & & & \\
AIC & $-2,5972$ & & & \\
SC & 23,5456 & & & \\
F-teste & & & & \\
(probab.) & $81,2305(0,00001)$ & & & \\
\hline
\end{tabular}

Nota: A hipótese de normalidade foi aceita ao nível de 1\%, quando utilizado o teste de Jarque-Bera. Contudo, a hipótese de homocedasticidade foi rejeitada quando utilizado o teste de BreuschPagan.

Pode-se observar que os sinais dos parâmetros estimados para as variáveis estão coerentes com o comportamento do mercado habitacional, pois há expectativas de que o consumo de habitação cresça com o aumento da renda, sugerindo que a habitação é um bem normal, e decresça quando o preço cresce. Também é razoável admitir que o indivíduo consome mais habitação se o prazo de financiamento é maior, pois ele pode fazer uma melhor programação das suas despesas no longo prazo. Em relação à poupança, que é uma variável representante do nível de riqueza da família, é natural que, sendo maior, o consumo também crescerá. Em relação ao sexo, não há nenhuma indicação na literatura sobre o sinal esperado, contudo esta análise coincide com outros estudos realizados (ver MAYO, 1981). Ou seja, as mulheres consomem mais habitação que os homens. A idade, contudo, apresenta sinal contrário do esperado: a teoria mostra que há uma tendência do indivíduo aumentar o seu padrão de consumo com a idade, até certo ponto, e depois haver um declínio. Uma possível explicação para este paradoxo pode estar associada a deficiências da amostra, que não traz informações suficientes para inferir este comportamento. Observa-se ainda que os coeficientes das variáveis ID e ID $^{2}$ não são significantes ao nível de $10 \%$. As demais variáveis se mostraram estatisticamente significantes ao nível de $1 \%$, com exceção do intercepto e da variável $\ln \mathrm{IPH}$. O modelo apresenta um razoável poder explicativo com coeficiente de determinação de 0,75. 
Pelos coeficientes estimados, pode-se concluir que: as elasticidades-renda e preço da habitação são 0,55 e $-0,23$, respectivamente; os mutuários do sexo feminino consomem em média $8,5 \%{ }^{16}$ mais habitação que os do sexo masculino; um acréscimo de $10 \%$ no prazo de financiamento gera um aumento de $0,65 \%$ no consumo de habitação e uma variação positiva de $10 \%$ na poupança do mutuário gera um acréscimo de $1,22 \%$ no consumo de habitação.

Apesar dos razoáveis resultados obtidos, deve-se esperar que, da mesma forma que a autocorrelação espacial está presente em diversas variáveis econômicas relacionadas com a demanda por habitação, o consumo de habitação também esteja autocorrelacionado espacialmente, uma vez que está associado à posição que ocupa no espaço. Desta forma, torna-se necessário realizar o diagnóstico da dependência espacial. Este diagnóstico foi realizado utilizando-se os testes LM Robusto (erro) e LM Robusto (defasagem) sobre os resíduos do modelo estimado na Tabela 6. Trabalhou-se com uma matriz de vizinhança W, com 194 linhas e 194 colunas, seguindo os mesmos procedimentos utilizados na seção anterior. A Tabela 7 apresenta os resultados para o diagnóstico realizado. O teste LM Robusto (defasagem) indica um forte efeito de defasagem espacial no consumo de habitação, uma vez que se mostrou significante a menos de $1 \%$, enquanto que, pelo teste LM Robusto (erro), a hipótese de autocorrelação nos resíduos é rejeitada. Ou seja, o efeito vizinhança parece estar afetando o consumo de habitação na cidade do Recife. Tabela 7 - Diagnóstico da Dependência Espacial da Demanda por Habitação no
Recife

\begin{tabular}{lccc}
\hline Teste & MI/GL & Valor & Probabilidade \\
\hline LM Robusto (erro) & 1 & 1,043571 & 0,306992 \\
LM Robusto (defasagem) & 1 & 19,576686 & 0,000010 \\
\hline
\end{tabular}

Diante dessa possibilidade de autocorrelação espacial entre os consumos de habitação ou efeito vizinhança, estima-se o Modelo de Defasagem Espacial de Demanda Habitacional, na forma funcional log-linear, é definido pela equação (12).

$$
\ln C H=f\left(\boldsymbol{W}_{-} \ln C H, \ln I P H, \ln R E, S, \rho, \beta\right)+\varepsilon
$$

onde $W_{-} \ln \mathrm{CH}$ é a variável explicativa espacialmente defasada do consumo de habitação, $\rho$ o seu coeficiente e as demais variáveis têm a mesma descrição do modelo (11).

16 Resultado de $\mathrm{e}^{0,08156}$. 
Os resultados da estimação empírica do modelo (12), que estão explicitados na Tabela 8 , indicam que o coeficiente $\rho$, da variável espacialmente defasada, $\mathrm{W} \_\ln \mathrm{CH}$, com valor 0,507 , é fortemente significante, o que é indicativo de uma grande probabilidade da existência do efeito de defasagem espacial na amostra. Ou seja, o consumo de habitação em determinado lugar da cidade é influenciado positivamente pelo consumo de habitação da vizinhança.

Tabela 8 - Resultados do Modelo Espacial de Demanda Habitacional para o Recife, com Intercepto

\begin{tabular}{lcccc}
\hline Variável & Coeficiente & Desvio Padrão & Estatística $z$ & Probabilidade \\
\hline$W \_$lnCH & 0,5070 & 0,0991 & 5,1159 & 0,0000 \\
Constante & 1,8047 & 1,8114 & 0,9962 & 0,3191 \\
$\operatorname{lnRE}$ & 0,5238 & 0,0294 & 17,7977 & 0,0000 \\
$\operatorname{lnIPH}$ & $-0,6822$ & 0,1822 & $-3,7426$ & 0,0002 \\
$S X$ & 0,0599 & 0,0339 & 1,7685 & 0,0769 \\
$I D$ & $-0,0033$ & 0,0018 & $-1,7897$ & 0,0734 \\
$I D^{2}$ & $5,68 \mathrm{E}-05$ & 0,0001 & 0,3940 & 0,6935 \\
$\ln P Z$ & 0,0611 & 0,0105 & 5,8178 & 0,0000 \\
$\ln P O$ & 0,1135 & 0,0119 & 9,4842 & 0,0000 \\
& & & & \\
$R^{2}$ & 0,7722 & & & \\
$A I C$ & $-20,3737$ & & & \\
$S C$ & 9,0370 & & & \\
\hline
\end{tabular}

Note-se que, embora os coeficientes das variáveis explicativas sejam significantes, com exceção da variável $\mathrm{ID}^{2}$, a hipótese do modelo passar pela origem é aceita, tendo em vista que o nível de significância do intercepto é superior a 30\%. Assim, um novo ajustamento sem o intercepto foi feito e se encontra na Tabela 9. Estes resultados confirmam, mais uma vez, a presença de fortes efeitos de autocorrelação espacial positiva entre os consumos de habitação, nos diversos pontos da cidade, dado que o coeficiente de defasagem espacial, $\rho$, com valor de 0,45 é significante a menos de $1 \%$. Assim, pode-se concluir que a demanda habitacional não depende somente das variáveis explicativas normalmente consideradas na literatura, tais como preço, renda e demais variáveis sociodemográficas da família, mas também é fortemente influenciada, de maneira positiva, pelo nível de demanda que ocorre na vizinhança. 
Tabela 9 - Resultados do Modelo de Defasagem Espacial para o Recife, sem Intercepto

\begin{tabular}{lcccc}
\hline Variável & Coeficiente & Desvio Padrão & Estatística z & Probabilidade \\
\hline$W \_l n C H$ & 0,449866 & 0,085398 & 5,26786 & 0,00000 \\
$\ln R E$ & 0,521328 & 0,029420 & 17,71979 & 0,00000 \\
$\ln I P H$ & $-0,503553$ & 0,025765 & $-19,54337$ & 0,00000 \\
$S X$ & 0,058059 & 0,033989 & 1,70817 & 0,08760 \\
$I D$ & 0,061953 & 0,010537 & 5,87917 & 0,00000 \\
$I D^{2}$ & $-0,003305$ & 0,001860 & $-1,77686$ & 0,07559 \\
$\ln P Z$ & $3,70 \mathrm{E}-05$ & 0,000143 & 0,25825 & 0,79621 \\
$\ln P O$ & 0,11283 & 0,011976 & 9,42062 & 0,00000 \\
$R^{2}$ & 0,77280 & & & \\
$A I C$ & $-21,4355$ & & & \\
$S C$ & 4,70736 & & & \\
\hline
\end{tabular}

Observa-se que houve modificações significativas nos resultados do modelo espacial de demanda habitacional (Tabela 9), quando comparados com os obtidos pelo modelo tradicional (Tabela 6), conforme resumo apresentado na Tabela 10.

Tabela 10 - Resultados do Modelo Tradicional (MT) e do Modelo Espacial (ME) para o Recife

\begin{tabular}{lccccc}
\hline \multirow{2}{*}{ Variável } & \multicolumn{2}{c}{ Coeficiente } & & \multicolumn{2}{c}{ Probabilidade } \\
\cline { 2 - 3 } \cline { 5 - 6 } & $\mathrm{MT}$ & $\mathrm{ME}$ & & $\mathrm{MT}$ & $\mathrm{ME}$ \\
\hline $\ln R E$ & 0,5522 & 0,5213 & & 0,0000 & 0,0000 \\
$\ln I P H$ & $-0,2354$ & $-0,5036$ & & 0,1768 & 0,0000 \\
$S X$ & 0,0816 & 0,0581 & & 0,0269 & 0,0876 \\
$I D$ & $-0,0032$ & $-0,0033$ & & 0,1084 & 0,0756 \\
$I D^{2}$ & 0,0001 & 0,0001 & & 0,7864 & 0,7962 \\
$\ln P Z$ & 0,0649 & 0,0620 & & 0,0000 & 0,0000 \\
$\ln P O$ & 0,1218 & 0,1128 & & 0,0000 & 0,0000 \\
\hline
\end{tabular}

Verifica-se que alguns coeficientes tiveram grandes alterações, tanto nas suas magnitudes, como nos seus níveis de significância. A maior delas se refere à elasticidade-preço da demanda, que passou de $-0,24$ no Modelo Tradicional, para - 0,50 no Modelo Espacial, representando uma variação de mais de 100\%. Também o nível 
de significância desta variável foi reduzido de 17\% no Modelo Tradicional, para um valor próximo de zero no Modelo Espacial, comprovando-se a existência de tendenciosidades e ineficiências no modelo tradicional, o que pode levar o pesquisador a conclusões equivocadas sobre os parâmetros da função de demanda habitacional estimados por este modelo.

Outro aspecto importante é que os valores obtidos para o modelo espacial pelos Critérios de Informação de Akaike (AIC) e Schwartz (SC) são bem inferiores aos encontrados no Modelo Tradicional, como se pode observar nos resultados constantes da Tabela 11, o que mostra a melhoria no modelo ajustado pela nova metodologia.

Tabela 11 - Comparação entre o Modelo Tradicional (MT) e o Modelo Espacial (ME)

\begin{tabular}{lcc}
\hline Critério & MT & ME \\
\hline AIC & $-2,60$ & $-21,43$ \\
SC & 23,54 & 4,70 \\
\hline
\end{tabular}

\section{Conclusões e Comentários Finais}

Pelos resultados encontrados neste trabalho, fica evidenciada a importância da utilização da econometria espacial nos estudos dos fenômenos relacionados à economia urbana, em particular na interpretação do comportamento do mercado habitacional. Nas análises empíricas realizadas com o objetivo de estimar uma Função de Demanda por Habitação para a cidade do Recife, com base em informações do Censo Demográfico (2000) e dados de imóveis financiados pela Caixa Econômica Federal, verificaram-se fortes indícios de dependência espacial em todas as variáveis econômicas exploradas, comprovando-se, desta forma, que somente os modelos espaciais podem fornecer estimativas confiáveis, caracterizadas pela não tendenciosidade, eficiência e consistência. A superioridade destes modelos em relação aos estimados pela Econometria Tradicional também foi comprovada pelos critérios de Akaike e Schwartz.

Verificou-se que a forma de considerar a questão espacial na equação de preços hedônicos, com base em distâncias da habitação a polos de influência ou dividindo o espaço em regiões através da inclusão de variáveis dummy, como vem sendo utilizada corriqueiramente na literatura (ver BRUECKNER, 1985; LUCENA, 1985; ERMISCH et al., 1996; TIWARE et al.,1999), não é capaz de explicar completamente as variações dos preços em relação à localização da habitação. Isto porque não 
são apenas os polos de influência que afetam a formação dos preços de mercado da habitação, mas existe uma verdadeira interação entre os dados amostrais, de forma que cada edifício funciona com um micropolo de influência sobre os seus vizinhos. Mostrou-se que, neste caso, o modelo de defasagem espacial fornece estimativas confiáveis para a equação de preços hedônicos, onde a variável de defasagem espacial, que leva em consideração todas as interações espaciais possíveis entre os dados, serve como proxy para variáveis locacionais não consideradas explicitamente. Também pode-se comprovar que as equações de demanda tradicionalmente estimadas, sem levar em conta os efeitos de dependência espacial, podem gerar resultados tendenciosos, onde a elasticidade-preço pelo Modelo Tradicional representa menos de $50 \%$ da estimativa realizada pelo Modelo Espacial, além de alterações significativas nas significâncias dos parâmetros, como o da elasticidade-preço que teve redução de 17\% (Modelo Tradicional) para um valor próximo de zero (Modelo Espacial) no seu nível de significância.

Do modelo espacial de preços hedônicos estimado, pode-se extrair várias informações de interesse, pois o conhecimento dos preços marginais que o consumidor está disposto a pagar pelas características da habitação pode ser a chave do sucesso para a implantação de novos empreendimentos. Por outro lado, o conhecimento das taxas de desvalorização com a idade e da taxa de valorização imobiliária é fundamental para os agentes financeiros que detêm o imóvel como garantia hipotecária nas operações de financiamento. Uma comparação da evolução do valor do imóvel no tempo e do saldo devedor é um parâmetro fundamental para o estudo da inadimplência.

A metodologia adotada para análise da dependência espacial do mercado habitacional do Recife pode ser aplicada para analisar o mercado habitacional em outras cidades, regiões, Estados ou países, com as devidas adaptações, pois há grande probabilidade da presença de efeitos de dependência espacial em qualquer mercado habitacional, pelas características próprias do bem habitação, principalmente em relação à fixação espacial.

Devido à grande probabilidade de dependência espacial entre as variáveis relacionadas com a demanda habitacional, pode-se concluir que as análises até então realizadas sobre o comportamento do mercado habitacional, tanto a nível de estimações de preços hedônicos como de estimações de elasticidades-preço e renda da demanda por habitação, sem levar em conta os efeitos de dependência espacial, podem apresentar conclusões equivocadas. 


\section{Referências}

ABNT. NBR-5676/90 - Norma para Avaliação de Imóveis Urbanos. São Paulo: ABNT, 1990.

AGUIRRE, A.; FARIA, D. M. C. P. A utilização de preços hedônicos na avaliação social de projetos. Belo Horizonte: Cedeplar/Face/UFMG, 1996. (Texto para Discussão, 103).

AGUIRRE, A.; MACEDO, P. B. R. Estimativa de preços hedônicos para o mercado imobiliário de Belo Horizonte. Anais do XVIII Encontro Brasileiro de Econometria. Águas de Lindóia, SP: ABE, 1996.

AKAIKE, H. Likelihood of a model and information criteria. Journal of Econometrics, v. 16, p. 3-14, 1981.

ANSELIN, L. Spatial econometrics: methods and models. Dordrecht: Kluwer Academic, 1988.

. Lagrange Multiplier Test Diagnostics for Spatial Dependence and Spatial Heterogeneity, Geographical Analysis, v. 20, p. 1-17, 1988a.

. SpaceStat: a program for the statistical analysis of spatial data, University of Illinois, Urbana-Champaign, IL,1990.

ANSELIN, L.; BERA, A. Spatial dependence in linear regression models with an introduction to spatial econometrics. In: ULLAH, A.; D. E. (Ed.). Handbook of applied economic statistics. Giles: Marcel Dekker, 1998.

ANSELIN L.; REY, S. Properties of tests for spatial dependence in linear regression models. Geographic Analysis, v. 23, p. 112-31, 1991.

BALARINE, O. F. O. Determinação do impacto de fatores sócio-econômicos na formação do estoque habitacional em Porto Alegre. Tese (Doutorado em Engenharia de Produção) - UFSC, Porto Alegre, 1995.

BIDERMAN, C. Forças de atração e expulsão na Grande São Paulo. São Paulo. Tese (Doutorado) - EASP/FGV, 2001.

BRUECKNER, J. Dynamic model of housing production. Journal of Urban Economics, v. 10, p. 1-14, 1985.

BURROUGH, P. A.; MCDONNELL, R. A. Principles of geographical information systems - spatial information systems and geostatistics. New York: Oxford University Press, 1998.

CLIFF, A. D.; ORD, J. K. Spatial processes - models and applications. London: Pion, 1981.

COOK Detection of influential observations in linear regression. Technometrics, v. 19, p. 15-18, 1977.

DANTAS, R. A. Avaliação de imóveis utilizando modelos especiais. In: CONGRESSO BRASILEIRO DE ENGENHARIA DE AVALIAÇÕES E PERÍCIAS, 9, 1997, São Paulo, 1997. 
DANTAS, R.A.; CORDEIRO, G.M. Uma nova metodologia para avaliação de imóveis utilizando modelos lineares generalizados. Revista Brasileira de Estatística, n. 191, p. 27-46, 1988.

. Evaluation of the Brazilian city of Recife's condominium market using generalized linear models The Appraisal Jornal, v. LXIX, n. 3, USA, 2001.

DANTAS, R. A.; ROCHA, F. S. Cómo Evitar Errores de Especificación en Ingeniería de Tasaciones I Congreso Internacional En Tasación Y Valoración. Valencia, Espanha: Ed. Universidad Politécnica de Valencia, 2002.

DIPASQUALE, D.; WHEATON, W. Housing market dynamics and the future of housing prices. Journal of Urban Economics, v. 35, 1994

ERMISCH, J. F., FINDLAY, J.; GIBB, K. The price elasticity of housing demand in britain issues of sample selection. Journal of Housing Economics, v. 5, p. 64-86, 1996.

FUNDAÇÃO JOÃO PINHEIRO Déficit habitacional no Brasil. Belo Horizonte, 2000.

GONZALEZ; M. A. S.; FORMOSO, C. T. Especificação de modelos de preços hedônicos para locação residencial em Porto Alegre. Cadernos IPPUR/UFRJ, v. 8, 1994.

GUERVÓS, R. C. Aproximación al valor de la vivienda: aplicación a la ciudad de Granada. Granada: Editorial Universidade de Granada, 1999.

HERMANN, B. M Estimando o preço implícito de amenidades urbanas: evidências para o município de São Paulo. Dissertação (Mestrado) - Departamento de Economia, Universidade de São Paulo, São Paulo, 2003.

IBGE. Boletim do SINAPI - Sistema Nacional de Pesquisa de Custos e Índices da Construção Civil. Rio de Janeiro: IBGE/CAIXA, jul. 2002.

LUCENA, J. M. P. O mercado habitacional no Brasil. Tese (Doutorado) - EPGE/ FGV-RJ, Rio de Janeiro, 1985.

MATHERON, G. Les variables regionalisées et leur estimation, Paris: Masson, 1965.

MAYO, S. K. Theory and estimation in economics of housing demand. Journal of Urban Economics, v. 10, p. 95-115, 1981.

OLMO, J. C. Teoriía de las Variables Regionalizadas - Aplicación en Economía Espacial y Valoración Inmobiliaria. Granada: Editorial Universidade de Granada, 1994.

PACE, R. K; BARRY, R.; SIRMANS, C. F. Spatial statistic and real state. Journal of Real State Finance and Economics, v. 17, n. 1, p. 5-13, 1998.

ROSEN, S. Hedonic prices and implicit markets: product differentiation perfect competition. Journal of Political Economy, v. 82, p 34-55, 1974. 
SANTOS, C.; CRUZ, B. A dinâmica dos mercados habitacionais metropolitanos: aspectos teóricos e uma aplicação para a Grande São Paulo. Brasília: IPEA, 2000. (Texto para Discussão, 713).

TIWARE, P.; PARICH, K.; PARIKH, J. Effective housing demand in Mumbai (Bombay) metropolitan region. Urban Studies, v. 36, n. 10, p. 1783-1809, 1999. 